\title{
Laparoscopically-assisted vaginal hysterectomy for enlarged uterus: operative outcomes and the learning curve
}

\author{
Mehmet Dolanbay, Mehmet S. Kutuk, Mahmut T. Ozgun, Semih Uludag, Yılmaz Sahin \\ Department of Obstetric and Gynecology, Erciyes University Faculty of Medicine, Kayseri, Turkey
}

\begin{abstract}
Objectives: The aim of the study was to compare the effects of uterine size and surgeon experience on the surgical outcomes of laparoscopically-assisted vaginal hysterectomy (LAVH) for benign gynecological conditions.

Material and methods: This was a retrospective analysis of $184 \mathrm{LAVH}$ cases. All hysterectomies were performed by the same surgeon and divided into two groups, with uterine weight of $<280 \mathrm{~g}$ (group 1) and uterine weight of $>280 \mathrm{~g}$ (group 2). The groups were compared in terms of the effects of the uterine size and surgeon experience vs. the operative outcomes (operative time, change in hemoglobin levels, hospital stay, and perioperative complications).

Results: No significant differences in mean age, parity, history of chronic systemic diseases and previous surgery history were observed between the two groups. However, operative time was significantly greater in group 2 as compared to group $1(132.1 \pm 42.7$ minutes vs. $111.5 \pm 30.4$ minutes, $\mathrm{p}<0.05)$. There were no differences in the hospital stay and perioperative complications between the two groups. One case of bladder injury occurred in each group and one patient underwent a second laparoscopic surgery for postoperative bleeding in group 2. Greater surgeon experience was demonstrated to be associated with decreased operative bleeding and, consequently, smaller differences between preoperative and postoperative hemoglobin levels. Operative time was also reduced as the surgeon was getting more experienced but the effect did not reach statistical significance.

Conclusions: Our study supports the thesis that LAVH is a safe and effective procedure for managing benign gynecological conditions. Despite increased operative time, LAVH can be safely performed for enlarged uterus in conjunction with increased surgeon experience.
\end{abstract}

Key words: laparoscopic assisted vaginal hysterectomy; large uterus; surgeon experience

Ginekologia Polska 2016; 87, 5: 333-337

\section{INTRODUCTION}

Hysterectomy is the most common gynecologic procedure in the developed countries [1]. Symptomatic uterine leiomyoma constitutes the main indication for hysterectomy. Also, it is applicable in lots of benign and malignant conditions such as endometrial hyperplasia and endometrial cancer. The route of hysterectomy depends on the technical and clinical factors, e.g. surgeon experience, uterine weight, previous surgery and vaginal delivery. Abdominal hysterectomy known as the "open" surgery ( $\mathrm{AH})$, and vaginal hysterectomy $(\mathrm{VH})$ are the dominant routes for that procedure. With the widespread use of laparoscopic surgery, the rate of laparoscopically-assisted surgery increased from $0.3 \%$ in 1990 to $14 \%$ in 2005 [2]. In parallel with the advancements in laparoscopic surgery and increased experiences with laparoscopically-assisted vaginal hysterectomy (LAVH), total laparoscopic hysterectomy (TLH) has become a common alternative to abdominal hysterectomy. The benefits of laparoscopic surgery, i.e. decreased pain, better cosmetic results, faster recovery, shorter hospital stay, and earlier return to normal activities increased the popularity of this route.

Despite the absence of a proven cut-off value for the uterine size above which VH is discouraged, conventional wisdom dictates the size of a gravid uterus $>12$ gestational 
weeks (> $280 \mathrm{~g}$ ) constitutes a relative contraindication to $\mathrm{VH}$ [3]. In these patients, LAVH may reduce the need for abdominal hysterectomy when the vaginal route alone is not feasible. Furthermore, LAVH can be safely performed in patients with history of previous surgery and adhesions, and possibility of adnexal pathology, which makes the VH a complex surgery. In contrast to abdominal or vaginal hysterectomy, LAVH needs extensive surgeon experience, longer operation time, and increased cost $[4,5]$.

In our study, we aimed to analyze the relation between uterine size and surgical outcomes (operative time, blood loss, and complication rates) in women who underwent LAVH for benign gynecological conditions. Secondly, we assessed the learning curve of a single surgeon with regard to these parameters and the overall surgical outcome.

\section{MATERIAL AND METHODS}

It was a retrospective analysis of patients who underwent LAVH for benign gynecological diseases at the Department of Obstetrics and Gynecology, University Hospital Erciyes, Kayseri, Turkey. We extracted their demographic and clinical data including age, parity, surgical indication, perioperative complications, operative time, change between pre- and postoperative hemoglobin, duration of the hospital stay, and uterine weight, retrospectively.

A total of 184 patients were included in the study. All operations were performed by the same surgeon (YS). The patients were subdivided into two groups on the basis of postoperative uterine weight. Group 1 consisted of women with uterine size of $<280 \mathrm{~g}$ ( $\mathrm{n}: 122$ ), and group 2 with uterine size of $>280 \mathrm{~g}$ ( $\mathrm{n}: 62)$. Written informed consent for participation in the study was obtained from all women.

\section{Surgical technique}

General anesthesia with endotracheal intubation was used and bladder catheterization was done with the patient lying in the dorsal lithotomic position before the uterine manipulator was inserted to the cervix. A 12-mm trocar was inserted to the umbilical area, followed by a $5-\mathrm{mm}$ trocar in each lower quadrant lateral to the inferior epigastric artery. A $10-\mathrm{mm}^{0}$ rigid camera was used. The liver, bowel, uterus and ovaries were checked carefully before start of the operation. Any adhesions between the uterus and the adnexa and the surrounding structures were divided by scissors. After bipolar coagulation and cutting of both round ligaments, the anterior peritoneum of the broad ligament was dissected toward the infundibulopelvic ligament. Then, the ovarian or infundibulopelvic ligaments were divided cautiously using bipolar cautery. The bladder was dissected from the lower uterine segment with the use of monopolar diathermy and sharp dissection by scissors. When the bleeding was controlled, the vaginal approach commenced. For the vaginal procedures, the anterior and the posterior aspects of the cervix were grasped with two strong tenaculum graspers and pulled downwards and outwards firmly. After making a vesicovaginal and rectovaginal space, a narrow Deaver retractor was placed in each of the spaces. The uterosacral-cardinal ligament complex is completely divided in one or two bites using a Wertheim, a long, heavy-toothed hysterectomy clamp (Aesculap, BJ 509,Germany) and tied on each side. The uterine arteries were clamped, cut, and tied singly on each side. The uterus was morcellated through the vaginal route. After hemostasis, the closure of the peritoneum was performed along with the closure of the vaginal vault. After the vaginal component of the operation, the abdominal cavity was re-insufflated with carbon dioxide and washed with warm saline solution. In some cases, drainage tube was inserted through the 5-mm trocar to remove the washing solution and gas.

The uteruses were weighed immediately after the extraction in the operation theatre using a standard weighing instrument. The operative time was defined as the time between the start of the skin incision and completion of the skin closure. Intraoperative bleeding was measured by extracting the postoperative hemoglobin value (measured at $24^{\text {th }}$ hours), from the preoperative value. Postoperative complications included bladder injury and reoperation for bleeding that occurred within three weeks after the operation.

The operations were encoded temporally according to the time they were performed (First operation was day 0 and the last one was day 1410), in order to analyze the effect of increased surgeon experience on blood loss and operative time. The relation between learning curve of the surgeon and the blood loss and operative time was statistically analyzed.

\section{Statistical analysis}

All data sets were subjected to normality tests using the Kolmogorov-Smirnov method and data were reported as either mean \pm standard deviation (mean \pm SD) (for normally distributed data and proportions), or as median with minimum and maximum (for skewed data). Comparison of variables between the two groups was made using the Mann-Whitney $U$ test. The effect of surgeon experience on operative time and blood loss was evaluated with Spearman's rank correlation coefficient. A two-tailed p-value of $<0.05$ was considered as statistically significant. Data were analyzed by Statistical package for the social sciences (SPSS) 15.0. (SPSS Inc., Chicago, USA).

\section{RESULTS}

A total of 904 hysterectomies for benign gynecological diseases were performed during the study period. Types of hysterectomy are shown in Table 1. One hundred and eighty-four women underwent LAVH for benign gyneco- 
logic conditions without any need for conversion to open surgery. Patient characteristics and indications for hysterectomies are presented in Table 2.

As shown in Table 2, there were no significant differences in terms of age, parity, history of chronic systemic diseases, and surgical history between the two groups. Half of patients in group 1 and 90\% in group 2 underwent LAVH

\section{Table 1. Types of hysterectomies during study period}

\begin{tabular}{|l|c|c|}
\hline Hysterectomy types & $\mathbf{n}$ & $\%$ \\
\hline Abdominal & 576 & 63.71 \\
\hline Vaginal & 144 & 15.92 \\
\hline Laparoscopic assisted vaginal & 184 & 20.35 \\
\hline
\end{tabular}

surgery due to uterine leiomyomas $(p<0.05)$. Dysfunctional uterine bleeding was significantly more common as a surgical indication in group $1(p<0.05)$

A comparison of surgical outcomes between the two groups is summarized in Table 3.

Mean uterine weight was $185.3(60-270) \pm 53.1 \mathrm{~g}$ and $416(280-770) \pm 117.8 \mathrm{~g}$ in the $<280 \mathrm{~g}$ and $>280 \mathrm{~g}$ groups, respectively $(p<0.05)$. There were no statistically significant differences in preoperative and postoperative hemoglobin levels, estimated blood loss, febrile morbidity, hospital stay, and perioperative complications between the two groups (Table 3 ). However, the operative time was significantly greater in group 2 as compared to group $1(132.1 \pm 42.7 \mathrm{~min}$. vs. $111.5 \pm 30.4$ min., $\mathrm{p}<0.05)$. There were one bladder injury

Table 2. The characteristics and operation indications of the groups of patients

\begin{tabular}{|c|c|c|c|}
\hline & $\begin{array}{c}\text { Group } 1 \\
\text { n (\%) }\end{array}$ & $\begin{array}{c}\text { Group } 2 \\
\text { n (\%) }\end{array}$ & $\mathbf{P}$ \\
\hline Age (years) & $46.17(34-65) \pm 5.34$ & $46.29(37-64) \pm 5.03$ & NS \\
\hline Parity & $3.55(0-8) \pm 1.43$ & $3.50(1-10) \pm 1.50$ & NS \\
\hline \multicolumn{4}{|l|}{ Previous abdominal surgery } \\
\hline Appendectomy & $6(4.91)$ & $2(3.22)$ & NS \\
\hline Caesarean section & $6(4.91)$ & $3(4.83)$ & NS \\
\hline Diabetes Mellitus & $5(4.09)$ & $4(6.45)$ & NS \\
\hline Hypertension & $5(4.09)$ & $3(4.83)$ & NS \\
\hline \multicolumn{4}{|l|}{ Hysterectomy indications } \\
\hline Leiomyoma & $62(50.81)$ & $56(90.32)$ & $<0.05$ \\
\hline Disfunctional uterine bleeding & $46(37.70)$ & $5(8.06)$ & $<0.05$ \\
\hline Endometrial hyperplasia & $13(10.65)$ & $1(1.61)$ & $<0.05$ \\
\hline Adenomyosis & $1(0.81)$ & 0 & NS \\
\hline
\end{tabular}

Values are mean (range) \pm SD or $n(\%)$. NS - not significant

Table 3. The comparison of surgical outcomes

\begin{tabular}{|l|c|c|c|}
\hline & Group 1 & Group 2 & P \\
\hline Uterine weight [g] & $185.3(60-270) \pm 53.1$ & $416(280-770) \pm 117.8$ & $<0.05$ \\
\hline Operating time (min) & $111.5(37-260) \pm 30.4$ & $132.1(60-300) \pm 42.7$ & $<0.05$ \\
\hline Preoperative Hb level [g/dL] & $12.1 \pm 1.3$ & $12.0 \pm 1.4$ & NS \\
\hline Postoperative Hb level [g/dL] & $10.5 \pm 1.4$ & $10.5 \pm 1.4$ & NS \\
\hline Perioperative Hb level change [g/dL] & $1.6 \pm 0.9$ & $1.5 \pm 1.1$ & NS \\
\hline Hospital stay (days) (range) & $1.33(1-2) \pm 0.4$ & $1.37(1-3) \pm 0.5$ & NS \\
\hline Perioperative complications & & & NS \\
\hline Bladder injury & 1 & 1 & NS \\
\hline Postoperative bleeding & 0 & 1 & NS \\
\hline Bowel injury & 0 & 0 & NS \\
\hline Major vessel injury & 0 & 0 & NS \\
\hline Urinary tract infection & 1 & 1 & \\
\hline
\end{tabular}

Values are mean (range) \pm SD. NS - not significant 
and one urinary tract infection in each group. One patient underwent a second laparoscopic surgery for postoperative bleeding in group 2. No major vessel, ureteral or bowel injuries occurred in either group. The effect of surgeon experience on the operative time and blood loss was evaluated. While increased surgeon experience was associated with remarkably less operative blood loss, shortening of the operative time was minimal and could not reach statistical significance.

\section{DISCUSSION}

Hysterectomy remains the mainstay of gynecologic surgery for uterine pathologies in the developed countries [6]. Although it is possible to perform hysterectomy in different ways, laparoscopic approaches have recently gained importance as an alternative to abdominal hysterectomy, especially in patients with a history of pelvic surgery, adhesion and concomitant ovarian mass $[6,7]$.

Our results showed that mean operative time was significantly longer for women with uterine weight of $>280 \mathrm{~g}$ than in the $<280 \mathrm{~g}$ group $(\mathrm{p}<0.05)$. Similarly, previous studies demonstrated uterine dimensions to determine the operative time $[8,4]$. In our experience, time allocated for morcellation of a bulky uterus was responsible for the difference between operative times in both investigated groups.

Despite the fact that vaginal hysterectomy is not the procedure of choice for bulky uteruses (> $280 \mathrm{~g}$ ), there is an ongoing debate as to whether LAVH can be an alternative route for these cases. Pelosi and Kadar reported a series including 20 patients whose uterus weighed $>500 \mathrm{~g}$, who underwent $\mathrm{LAVH}$, and only one case of bleeding requiring blood transfusion [9]. Chang et al., compared surgical outcomes between large and small uteruses, with $750 \mathrm{~g}$ as the cut-off point, and found that the operative time, blood loss, and transfusion requirement, were significantly increased in the large uterus group. These authors concluded that higher blood loss and operative time were acceptable for these patients when the other factors (short hospital stay, decreased wound infection, and analgesic dosage) were taken into account. However, by applying various combinations of the special strategies, such as inserting the trocars more cephalad than usual in order to avoid direct puncture into the bulky uterus, myomectomy with the aid of a myoma screw, may also be valuable in overcoming the limited operation field, to avoid ureter or bladder injury and to facilitate subsequent vaginal hysterectomy [10].

In our study, while increased surgeon experience was associated with remarkably less operative blood loss, shortening of the operative time was minimal and could not reach statistical significance. In addition, statistical analysis revealed that improvement in intraoperative blood loss was observed after 25 surgeries. Altgassen et al., demonstrated the minimum number of operations before surgical complications of LAVH decreased to be 30 [11]. Ou et al., in their multicenter study including 839 LAVH cases, demonstrated lack of correlation between surgeon experience and operative time [12]. It can be speculated that, the more experienced the surgeon becomes, the more complicated case $s /$ he attempted to perform, and therefore, the operative time did not change markedly.

In our study, the total complication rate was $2.71 \%$. LAVH was successfully performed in 184 patients, and only one relaparoscopy was needed in a patient from group 2 due to early postoperative hemorrhage requiring blood transfusion. Massive hemoperitoneum was detected and approximately $1000 \mathrm{~mL}$ blood were removed during the operation. Bleeding originated from the right infundibulopelvic ligament and was kept down with bipolar coagulation.-Bladder injuries occurred during sharp dissection of the bladder from the low uterine segment during the laparoscopic part of the operation. The lacerations were little than $2 \mathrm{~cm}$ and were repaired laparoscopically by suturing bladder mucosa and serosa separately with absorbable sutures. Bladder catheters remained in place for 5 days. Other authors demonstrated surgical complication of LAVH to be similar between uteruses weighing $>500 \mathrm{~g}$ and $<$ than $500 \mathrm{~g}$ $[4,8]$, and reported an overall surgical complication rate of $8.7 \%$ [8], what is consistent with our findings. Our results indicate that the complication rates between the two groups were comparable. Therefore, $\mathrm{LAVH}$ seems to be a reasonable option for patients with large uteruses.

While increased surgeon experience was associated with remarkably less operative blood loss, the shortening of operative time was minimal and could not reach statistical significance. Our study demonstrates that LAVH is a safe and effective procedure to manage benign gynecological conditions and, despite increased operative time, LAVH can be safely performed for enlarged uterus in conjunction with increased surgeon experience.

\section{Conflict of interest}

No conflict of interest was declared by the authors.

\section{Financial Disclosure}

The authors declared that this study has received no financial support.

\section{REFERENCES}

1. Taylor SM, Romero AA, Kammerer-Doak DN, [et al.]. Abdominal hysterectomy for the enlarged myomatous uterus compared with vaginal hysterectomy with morcellation. Am J Obstet Gynecol. 2003, 189 (6): 1579-1582; discussion 1582-1583.

2. Jacoby VL, Autry A, Jacobson G, [et al.]. Nationwide use of laparoscopic hysterectomy compared with abdominal and vaginal approaches. Obstet Gynecol. 2009, 114 (5): 1041-1048.

3. American College of Obstetricians and Gynecologists. Quality assurance in obstetrics and gynecology. Washington, DC: The College, 1989. 
4. Wang $\mathrm{CJ}$, Yen $\mathrm{CF}$, Lee $\mathrm{CL}$, [et al.]. Laparoscopically assisted vaginal hysterectomy for large uterus: a comparative study. Eur J Obstet Gynecol Reprod Biol. 2004, 115 (2), 219-223.

5. Doucette RC, Scott JR. Comparison of laparoscopically assisted vaginal hysterectomy with abdominal and vaginal hysterectomy. J Reprod Med. 1996, 41 (1), 1-6.

6. Hobson DT, Imudia AN, Al-Safi ZA, [et al.]. Comparative analysis of different laparoscopic hysterectomy procedures. Arch Gynecol Obstet. 2012, 285 (5), 1353-1361.

7. Song T, Kim TJ, Kang H, [et al.]. Factors associated with complications and conversion to laparotomy in women undergoing laparoscopically assisted vaginal hysterectomy. Acta Obstet Gynecol Scand. 2012, 91 (5), 620-624.
8. Ark C, Güngördük K, Celebi I, [et al.]. Experience with laparoscopic -assisted vaginal hysterectomy for the enlarged uterus. Arch Gynecol Obstet. 2009, 280 (3), 425-430.

9. Pelosi MA, Kadar N. Laparoscopically assisted hysterectomy for uteri weighing $500 \mathrm{~g}$ or more. J Am Assoc Gynecol Laparosc. 1994, 1 (4 Pt 1), 405-409.

10. Chang WC, Huang SC, Sheu BC, [et al.]. LAVH for large uteri by various strategies. Acta Obstet Gynecol Scand. 2008, 87 (5), 558-563.

11. Altgassen $C$, Michels W, Schneider A. Learning laparoscopic-assisted hysterectomy. Obstet Gynecol. 2004, 104 (2), 308-313.

12. Ou CS, Beadle E, Presthus J, [et al.]. A multicenter review of 839 laparoscopic-assisted vaginal hysterectomies. J Am Assoc Gynecol Laparosc. 1994, 1 (4 Pt 1), 417-422. 